\title{
Traitement par ultrasons focalisés du cancer localisé de la prostate : Résultats carcinologiques et pronostic sexuel
}

\author{
Albert GELET1, Laura POISSONNIER1, Jean-Yves CHAPELON2, Raymonde BOUVIER1, \\ Olivier ROUVIERE1, Denis LYONNET1, Jean Michel DUBERNARD1. \\ 1 Hôpital E. Herriot, 2 Unité INSERM 556, LYON
}

\section{RESUME}

Objectifs : Evaluer l'efficacité du traitement du cancer localisé de la prostate par ultrasons focalisés de haute intensité (HIFU), et son retentissement sur la fonction sexuelle.

Matériels et méthodes : 120 patients potentiellement curables présentant un cancer de la prostate de stade clinique T1-T2 avec un PSA initial $<10$ $\mathrm{ng} / \mathrm{ml}$ (groupe 1) et 167 patients avec un PSA initial $<30 \mathrm{ng} / \mathrm{ml}$ (groupe 2), non candidats à la prostatectomie radicale, ont été traités par HIFU (ABLATHERM $^{\circledR}$, EDAP SA). Dans ces 2 populations, l'échec clinique a été défini comme la nécessité de recourir à un traitement adjuvant. La progression de la maladie (échec biologique) a été strictement définie par l'identification d'un cancer résiduel sur les biopsies de contrôle (quelque soit le taux de PSA) ou par 3 élévations successives du PSA (lorsque les biopsies de contrôle étaient négatives) avec une vélocité supérieure à $0,75 \mathrm{ng} / \mathrm{ml} / \mathrm{an}$. Les taux de survie sans progression ont été calculés selon la méthode de Kaplan-Meier. Les taux de succès stratifiés selon les facteurs de risque ont été comparés par Log-Rank tests. L'impact du traitement sur la fonction sexuelle a été évalué au moyen d'un questionnaire chez 70 patients ayant reçu un traitement standard et 28 patients pour lesquels le traitement tentait de préserver les nerfs érecteurs.

Résultats : Le suivi moyen est de 27 mois et 23 mois dans les groupes 1 et 2 , respectivement. Les biopsies de contrôle ne montrent pas de cancer résiduel chez 103 patients du groupe 1 (86\%), et
131 patients du groupe 2 (78\%). Dans le groupe 1 , un cancer résiduel a été identifié chez 17 patients mais seuls 6 patients ont nécessité un traitement adjuvant (hormonal : $n=2$, radiothérapie : $n=4$ ), soit un taux de succès clinique de $95 \%$. Dans le groupe 2, 36 patients ont présenté des biopsies positives lors du suivi, dont 21 ayant nécessité un traitement adjuvant (hormonal : $n=10$, radiothérapie : $n=11$ ), soit un taux de succès clinique de $87,5 \%$. Le taux de survie sans progression est de $76,9 \%$ et $66 \%$ dans les groupes 1 et 2, respectivement. Dans le groupe 2, les taux de survie sans progression ont été stratifiés en fonction du niveau de risque initial : $85 \%$ pour les patients à faible risque, $67,5 \%$ pour les patients à risque intermédiaire, et $42 \%$ pour les patients à haut risque. Dans la population globale, 70 patients avaient une fonction sexuelle normale avant traitement : 25 patients (36\%) ont conservé des érections autorisant les rapports sexuels après le traitement par ultrasons. Une procédure de traitement visant à épargner les nerfs érecteurs a été testée sur 28 patients : $43 \%$ de ces patients ont conservé des érections permettant la pénétration vaginale, montrant ainsi que cette procédure est encore perfectible.

Conclusion : Ces résultats montrent que le traitement par ultrasons focalisés est une option thérapeutique dont les résultats carcinologiques sont

\section{Correspondance :}

Dr Albert GELET - Urologie et Chirurgie de la transplantation. Hôpital Edouard Herriot, 69437 Lyon -

Tel 33.4.72.11.05.83 - Fax 33.4.72.11.05.59 -

Email albert.gelet@chu-lyon.fr 
comparables aux autres traitements non chirurgicaux du cancer de la prostate. Après traitement complet de la glande prostatique, plus d'un tiers des patients conservent une érection permettant la pénétration vaginale, ce résultat étant à interpréter pour une population âgée de 72 ans en moyenne. La mise au point d'une procédure permettant d'épargner les nerfs érecteurs est en cours.

Mots clés : ultrasons focalisés, cancer localisé de prostate, dysérection

\section{INTRODUCTION}

Un projet de recherche concernant la destruction des tumeurs cancéreuses par ultrasons focalisés a été initié en 1989 à Lyon grâce à la collaboration de 3 partenaires : l'unité INSERM 556 (Institut National pour la Santé Et la Recherche Médicale), le service d'urologie de l'Hôpital Edouard Herriot et la société EDAP SA.

Après une expérimentation sur l'animal $[5,6,16]$ et des essais cliniques réalisés à partir de 1993 [17], ce projet a abouti à la commercialisation d'un appareil appelé ABLATHERM $^{\circledR}$ destiné au traitement des cancers localisés de la prostate décelés chez des patients qui ne relèvent pas de la chirurgie à cause de leur âge et de leur état général (ou qui la refusent à cause de sa morbidité spécifique). L'objectif était de pouvoir proposer à ce type de patients un traitement aussi peu invasif que possible. L'homologation Européenne de l'appareil a été obtenue en juin 1999 (marquage $\mathrm{CE}$ ) et 10 centres sont actuellement équipés de ce matériel.

L'objectif de l'étude est de rapporter les résultats carcinologiques du traitement par ultrasons focalisés et d'apprécier le retentissement sexuel de cette nouvelle approche thérapeutique.

\section{MATERIEL ET METHODES}

\section{Principe}

Un faisceau d'ultrasons convergents de haute intensité est émis par un transducteur piézo-électrique focalisé. Les ultrasons sont émis par salves qui durent plusieurs secondes. A l'endroit où les ultrasons se concentrent (point focal), l'absorption soudaine et intense du faisceau ultrasonore crée une élévation brutale de la température (entre 85 et $100^{\circ} \mathrm{C}$ ) qui détruit les cellules situées dans la zone cible. La zone cible détruite par chaque tir est ellipsoïdale et mesure 19 à $24 \mathrm{~mm}$ de haut par $2 \mathrm{~mm}$ de diamètre. Le mécanisme qui provoque la destruction cellulaire est en fait une combinaison de 2 facteurs : l'absorption des ultrasons au point focal d'une part et, d'autre part, un phénomè- ne de cavitation contrôlée qui se produit en arrière du point focal et qui contribue à augmenter la hauteur de la lésion élémentaire créée par chaque tir. En répétant les tirs et en déplaçant le point focal entre chaque tir, il est possible de détruire un volume.

Un troisième phénomène intervient alors : l'accumulation thermique.

Ce phénomène permet d'expliquer pourquoi la zone de nécrose de coagulation observée après plusieurs tirs est de taille plus importante que la zone de nécrose théorique calculée en juxtaposant les lésions élémentaires crées par chaque tir. En effet, pour un tir, le faisceau ultrasonore est focalisé en un point et l'énergie apportée est transformée en chaleur selon une forme ellipsoïdale. Au moment du tir suivant, effectué après mouvement du transducteur, le faisceau ultrasonore est focalisé sur un autre point, proche du précédent, et la lésion se propage de la même façon. Mais si la localisation des tirs successifs est suffisamment proche, certaines cellules concernées par le deuxième tir présentent une température plus élevée que la température de base, du fait de la chaleur diffusée par le premier tir. La dose thermique reçue par ces cellules est donc modifiée par la proximité des tirs et la forme de la lésion finale n'est pas limitée à la zone des tirs. Ce phénomène d'accumulation thermique est observé lorsque les tissus touchés par un premier tir n'ont pas le temps de revenir à la température initiale avant le tir suivant. Une durée courte du temps de repos entre chaque tir permet donc d'obtenir une lésion plus étendue. C'est ce qui est réalisé avec l'ABLATHERM ${ }^{\circledR}$, avec des délais de 5 secondes entre chaque tir. Ce processus a été modélisé [9] et il est donc possible de calculer les dimensions de la zone de nécrose en fonctions des paramètres de traitement (intensité acoustique, durée de chaque tir, espacement entre les tirs, nombre de tirs, géométrie de la cible) : schématiquement, la lésion obtenue est trapézoïdale et "déborde" le volume cible latéralement de 6 à $8 \mathrm{~mm}$, ce qui est pris en compte lors du planning de thérapie.

\section{Matériel}

L'ABLATHERM $^{\circledR}$ est un appareil qui intègre plusieurs composantes :

a) Un support sur lequel repose le patient pendant le traitement.

b) Un générateur d'ultrasons.

c) Un échographe qui permet la localisation de la prostate par le chirurgien.

d) Une tête de tir endorectale qui combine une sonde d'imagerie biplan et le transducteur de traitement qui émet les ultrasons focalisés. Ces deux éléments sont placés dans un ballon de latex rempli d'un liquide anticavitation. 
e) Un ordinateur qui contrôle et dirige les tirs selon les instructions données par le chirurgien.

De nombreux dispositifs de sécurités sont intégrés à l'appareil. Les plus importants sont d'une part, un contrôle permanent de la position du transducteur de traitement par rapport à la paroi du rectum, d'autre part, un détecteur de mouvement du patient.

Ces systèmes arrêtent automatiquement les tirs si une anomalie est détectée en cours de traitement (déplacement de la prostate et/ou du patient au cours du traitement).

Enfin un système de refroidissement maintient la température de la muqueuse rectale entre 12 et $14^{\circ} \mathrm{C}$.

\section{Sélection des patients}

Les indications du traitement par ultrasons ont été définies à l'issue du marquage CE. Ce traitement s'adresse en premier lieu aux patients porteurs d'un cancer localisé T1, T2, non candidats à la prostatectomie (en raison de leur âge ou de leur état général) et en second lieu, aux patients qui sont de mauvais candidats à la chirurgie (stade T1b, stades T2b et $\mathrm{T} 2 \mathrm{c}$ ).

\section{Réalisation du traitement}

Le patient est hospitalisé la veille du traitement, une préparation digestive analogue à celle réalisée pour une coloscopie est effectuée. Le traitement se déroule sous anesthésie la plus souvent loco-régionale (rachianesthésie). Depuis mars 2000 , nous réalisons une résection endoscopique du tissu adénomateux en début d'anesthésie : ce geste réduit la durée du sondage post opératoire et fait pratiquement disparaître les phénomènes d'obstruction par élimination de débris nécrotiques (sloughing) observés avant cette modification technique.

Le traitement par ultrasons est ensuite effectué en décubitus latéral droit (patient couché en chien de fusil sur le côté droit). Une stricte immobilité est nécessaire pendant le traitement.

Le couplage entre le ballon et le rectum est assuré par du gel échographique.

Le chirurgien introduit la tête de tir dans le rectum et remplit le ballon de latex stérile avec $150 \mathrm{ml}$ de liquide de couplage anticavitation, qui est maintenu à température constante pendant toute la durée du traitement. Il faut ensuite placer la tête de tir en position d'imagerie.

Le chirurgien repère les limites de la prostate dans le plan sagittal, et définit sur l'écran de contrôle la zone qu'il souhaite traiter. Le traitement se déroule en plusieurs séquences ( 2 à 5 ) selon le volume de la prostate. Pour chaque tranche de prostate, l'opérateur définit les limites droite et gauche de la zone à traiter. Les lésions programmées appa- raissent sur l'écran de contrôle comme une rangée d'ellipses. Le logiciel de l'appareil détecte la paroi rectale et aligne automatiquement les tirs le long de la capsule postérieure de la prostate en laissant une marge de sécurité par rapport à la paroi rectale.

Quand les coordonnées tridimensionnelles de la zone sont ainsi définies, le logiciel rétracte la sonde d'échographie biplan et place le transducteur de thérapie parallèlement à la face postérieure du rectum. Le traitement se déroule par couches successives de $1,7 \mathrm{~mm}$ d'épaisseur régulièrement disposées de l'apex vers la base. Habituellement, 5 volumes de traitement sont successivement définis : apex, partie moyenne et base d'un lobe, puis partie moyenne et base controlatérales (400 à 600 tirs sont généralement effectués). Selon l'objectif du traitement, le chirurgien peut à volonté pour chaque volume cible suivre strictement les contours de la prostate, ou préserver une marge de sécurité entre le dernier tir et la capsule prostatique pour éviter de léser les bandelettes neuro-vasculaires, ou au contraire étendre la zone cible au-delà de la capsule pour nécroser volontairement la graisse péri prostatique en cas de tumeur localement évoluée (T2b,T3a).

Le traitement dure de 1 à 2 heures selon le volume à traiter. Lorsque le traitement est fini, le chirurgien met en place une sonde à demeure $20 \mathrm{Fr}$ double courant (un cathéter sus pubien est mis en place chez les patients qui ont subi une adénomectomie dans les mois ou les années précédant le traitement ABLATHERM ${ }^{\circledR}$ ). En général, le patient reprend ses mictions au troisième jour post opératoire et regagne son domicile le lendemain.

\section{Suivi post opératoire}

La zone de nécrose peut être visualisée dès la $48^{\mathrm{ème}}$ heure par IRM avec injection de gadolinium : la zone traitée ne prend pas l'agent de contraste car elle est totalement dévascularisée mais cet examen n'est utilisé que dans le cadre de protocole de recherche [35].

Le taux de PSA s'élève brutalement 24 heures après le traitement ( 2 à 7 fois le taux de base), puis il redescend progressivement et le point nadir est obtenu entre 3 et 5 mois après la session.

Les biopsies de contrôle montrent des lésions de nécrose de coagulation pendant les 3 mois qui suivent la session, puis la fibrose apparaît progressivement.

C'est en fonction du bilan à 3 mois (PSA et biopsies), qu'une seconde séance de traitement peut être décidée, dans des conditions identiques à celles précédemment décrites : le meilleur moment pour la réaliser est le 6ème mois, la rétraction du volume prostatique étant alors maximum. 


\section{Analyse des résultats}

Pour chaque patient, la durée du suivi a été définie comme l'intervalle de temps entre la dernière séance HIFU et le dernier dosage de PSA réalisé. Les taux de survie sans progression ont été déterminés selon la méthode de Kaplan-Meier, l'évènement progression étant strictement défini comme toute biopsie positive (quelque soit le taux de PSA) ou 3 augmentations successives du taux de PSA avec une vélocité supérieure ou égale à $0,75 \mathrm{ng} / \mathrm{ml} / \mathrm{an}$ pour les patients présentant des biopsies négatives ; cette vélocité du PSA est la valeur seuil proposée par Pearson et Carter [26] pour les patients présentant un cancer localisé de la prostate. Les taux de survie sans progression ont été comparés grâce au Log-rank test avec $\mathrm{p}<0,05$ considéré comme seuil de signification statistique.

Le suivi de la fonction urinaire et de la fonction sexuelle a été réalisé par questionnaires auto-administrés. En ce qui concerne la fonction érectile pour les patients traités avant 2002, c'est le critère de la pénétration vaginale qui a été simplement considéré (possible/pas possible) ; pour les patients traités en 2002, nous avons utilisé les questions concernant la qualité de la fonction érectile du questionnaire IIEF.

\section{RESULTATS}

\section{Résultats carcinologiques des patients inclus avec un PSA initial $<10 \mathrm{ng} / \mathrm{ml}$}

Depuis 1993, 400 patients ont été traités en première intention pour cancer localisé de la prostate à l'hôpital E Herriot. Nous avons étudié les résultats à long terme de 120 patients dont le PSA initial était inférieur à $10 \mathrm{ng} / \mathrm{ml}$ (donc considérés potentiellement curables) et qui ont été tous évalués par des biopsies de contrôle et par le suivi du PSA.

Les caractéristiques principales de ces patients étaient : un âge moyen de 71,2 $\pm 5,34$ ans; un stade clinique $\mathrm{T} 1$ pour $\mathrm{n}=61$ et $\mathrm{T} 2$ pour $\mathrm{n}=59$; un PSA initial moyen de $5,67 \pm$ $2,47 \mathrm{ng} / \mathrm{ml}$; un score de Gleason de $2-6$ pour $\mathrm{n}=77$ et de 7 10 pour $n=43$, et un volume prostatique moyen de $33,6 \pm$ $16,5 \mathrm{ml}$. Le suivi moyen des patients est de 27 mois (extrêmes : 3-96 mois).

Aucun foyer cancéreux résiduel n'a été décelé par les biopsies de contrôle chez 103 patients $(86 \%)$ et 90 patients (75\%) ont un PSA nadir $<0,5 \mathrm{ng} / \mathrm{ml}$. Le taux d'échec clinique défini par la nécessité d'un traitement adjuvant est de $5 \%$ ( 6 patients): 4 patients ont reçu une radiothérapie externe adjuvante (sans effet indésirable particulier) et 2 patients ont été placés sous traitement hormonal. Les 11 autres patients présentant de petits foyers cancéreux résiduels sont simplement surveillés car leur taux de PSA est bas (PSA moyen $0,85 \pm 0,78 \mathrm{ng} / \mathrm{ml}$ ) et très lentement ascendant : une séance complémentaire d'ultrasons ne sera réalisée que si l'élévation du taux de PSA le justifie.

Nous avons défini la survie sans progression comme l'association de biopsies de contrôle négatives avec un PSA stable : sont considérés en échec tous les patients présentant un foyer cancéreux résiduel sur les biopsies de contrôle (quelque soit la valeur du PSA), et tous les patients présentant un taux de PSA ascendant (pour les patients avec biopsies de contrôle négatives). En utilisant ces critères très restrictifs, le taux de survie sans progression (Disease Free) pour l'ensemble des 120 patients est de $77 \%$ à 50 mois (Figure 1). Le taux de survie sans progression s'élève à $85 \%$ chez les patients porteurs de cancer bien différenciés (Gleason 2-6) versus $61 \%$ chez les patients porteurs de cancers peu différenciés (score de Gleason 7-10), cette différence est statistiquement significative (Figure 2).

Pour l'ensemble des patients traités (incluant les patients traités avec les prototypes entre 1993 et 1998), les principales complications ont été les scléroses cervico-prostatiques (18\%) et l'incontinence d'effort $(18,6 \%$, évaluée par questionnaire) : grade 1 (1 garniture/jour) : 17 (14\%), grade 2 ( $>1$ garniture/jour) : 4 (3\%), grade 3 (incontinence totale) : $2(1,6 \%)$.

Depuis janvier 2000 (mise en fonction de l'appareil ABLATHERM ${ }^{\circledR}$ "de série" combinée à la réalisation d'une résection endoscopique associée systématique), la durée moyenne de cathétérisme post opératoire est de 6 jours (versus 13 jours auparavant), le taux de sténose est descendu à $9 \%$. Aucune incontinence sévère (grade 3 ) n'est survenue et le taux d'incontinence d'effort s'est réduit (incontinence grade $1: 11 \%$, grade $2: 2 \%$ ).

\section{Résultats carcinologiques des patients inclus avec un PSA initial $<30$ ng/ml}

167 patients correspondant à ce critère d'inclusion sont évaluables par biopsies de contrôle et par suivi du PSA : les caractéristiques principales de ces patients sont un âge moyen de $71,8 \pm 5,11$ ans ; un stade clinique $\mathrm{T} 1$ pour $\mathrm{n}=77, \mathrm{~T} 2$ pour $\mathrm{n}=85$ et $\mathrm{T} 3$ pour $\mathrm{n}=5$; un PSA initial de 9,3 $\pm 6,01 \mathrm{n} / \mathrm{ml}$; un score de Gleason de 2-6 pour $\mathrm{n}=98,7$ pour $n=44$ et $8-10$ pour $n=25$, et un volume prostatique moyen de $34,4 \pm 16,69 \mathrm{ml}$.

Le suivi moyen des patients est de 23 mois (extrêmes : 390 mois). $78 \%$ des patients ont des biopsies de contrôle négatives et $69 \%$ (115 patients) ont un PSA nadir $<0,5$ $\mathrm{ng} / \mathrm{ml}$. Le taux d'échec clinique défini par la nécessité d'un traitement adjuvant est de 12,5\% (21 patients): 11 patients ont reçu une radiothérapie externe adjuvante et 10 patients ont été placés sous traitement hormonal.

Le taux de survie sans progression calculé pour l'ensemble des 167 patients avec les mêmes critères que pour l'étude précédente sont de $66 \%$ à 50 mois (Figure 3). 


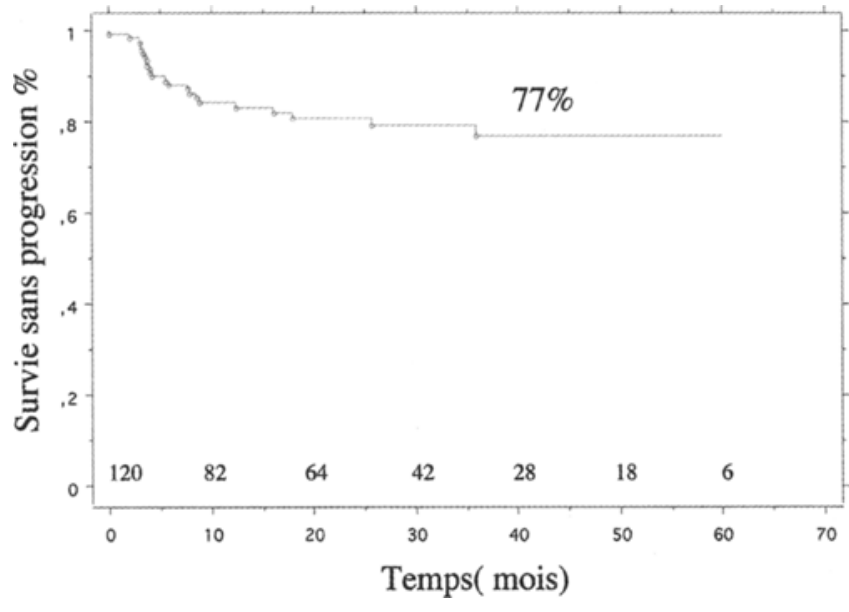

Figure 1 : Survie sans progression chez 120 patients ayant un PSA initial $<10$ ng.

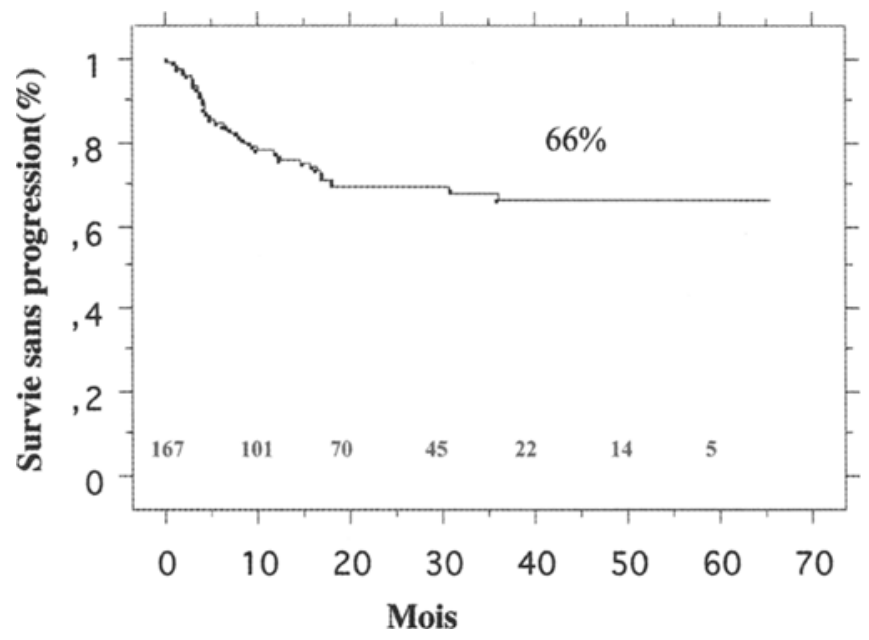

Figure 3 : Survie sans progression chez 167 patients.

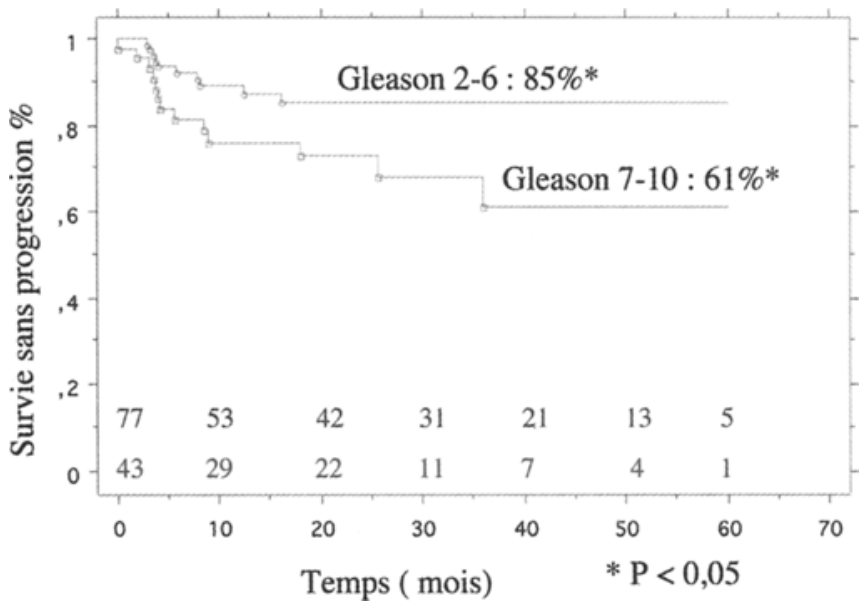

Figure 2 : Survie sans progression selon le score de Gleason.

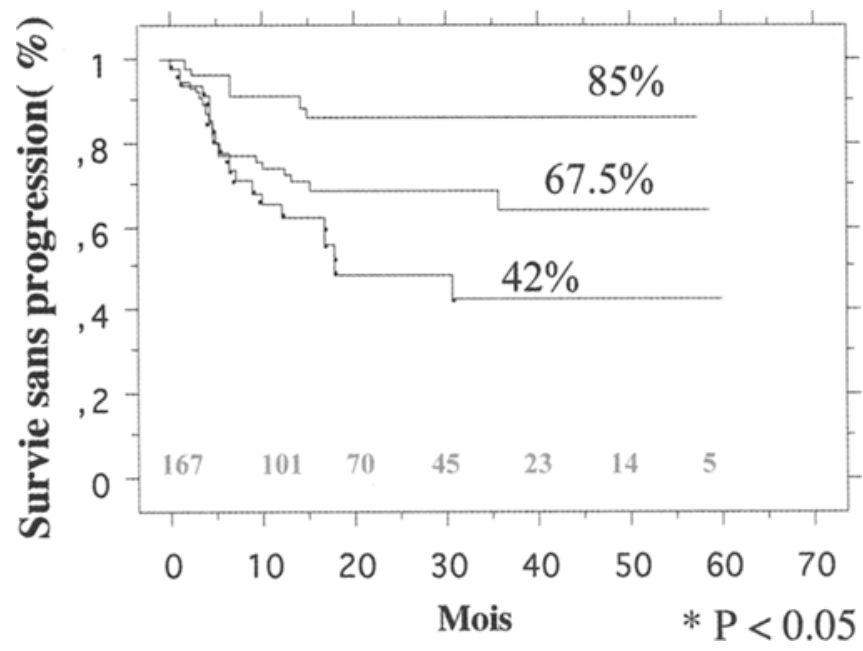

Figure 4 : Survie sans progression en fonction des groupes à risques 
Le taux de survie sans progression est corrélé de façon significative aux facteurs de risques définis par D'Amico, à savoir : patients à bas risque : T1 - T2a et PSA $<10$ et Gleason 2-6, patients à risque intermédiaire : T2b ou PSA 10-20 ou Gleason 7, et patients à haut risque : T2c ou PSA $>20$ ou Gleason 8-10.

Les taux de survie sans progression à 50 mois pour les patients à bas risque, à risque intermédiaire et à haut risque sont respectivement de $85 \%, 67,5 \%$ et de $42 \%$ (Figure 4 ).

\section{Résultats sur la fonction sexuelle}

Etant donné que le tissu prostatique est détruit, tous les patients présentent une disparition de l'éjaculation : seule la préservation de la fonction érectile peut donc être analysée.

Le taux de disparition des érections évalué par questionnaire simple (oui/non) chez 70 patients qui avaient une fonction sexuelle conservée et qui ont été traités sans précautions particulières entre 1993 et 2001 a été de $64 \%$ (ce résultat est à interpréter en tenant compte de l'âge moyen des patients : 72 ans).

Une étude complémentaire a été réalisée en 2002 chez 28 patients dont la fonction érectile était conservée et qui souhaitaient un traitement préservant la fonction sexuelle : dans ce groupe de patients, le planning de traitement comportait une marge de sécurité de $6 \mathrm{~mm}$ entre le dernier tir réalisé et la capsule prostatique sur une hauteur de $20 \mathrm{~mm}$ à partir de l'apex : au-delà (partie moyenne et base), le traitement était étendu jusqu'à la capsule. Pour les patients de cette étude, la fonction érectile a été évaluée par le questionnaire IIEF entre 3 et 6 mois après la session HIFU : la fonction érectile a été préservée plus ou mois complètement chez 12 patients (43\%) autorisant la pénétration vaginale, 6 patients présentaient une altération légère de l'érection (score > 17), 3 une altération modérée (score entre 11 et 17 ) et 3 une altération sévère (score < 11). Chez tous les autres patients $(57 \%)$, la pénétration vaginale était impossible.

\section{DISCUSSION}

\section{Efficacité carcinologique}

Pour démontrer l'efficacité d'un traitement sur le cancer de la prostate, il faut en théorie démontrer que ce traitement permet d'obtenir un prolongement de la durée de vie du patient (survie spécifique). Compte tenu de l'évolution très lente du cancer de prostate, un recul de 15 ans est nécessaire. Cependant, grâce à l'évolution du PSA et aux biopsies de contrôle, on peut estimer plus tôt le bénéfice qui va être procuré par le traitement (survie sans progression). Il est possible de comparer les résultats des traitements par agents physiques entre eux tout en sachant que la plupart des études reposent uniquement sur le suivi du PSA : en ce qui concerne les patients traités par radiothérapie externe, le taux de survie sans progression biochimique (critère ASTRO) varie de $75 \%$ à $98 \%$ selon les séries pour les patients dont le PSA initial est inférieur à $10 \mathrm{ng} / \mathrm{ml}$ et avec un score de Gleason maximum de $6[15,12,19,31,41,48$, $22,13,14,28,38,30,50,32]$. En ce qui concerne les patients traités par curiethérapie, le taux de survie sans progression varie de $57 \%$ à $92,6 \%$ pour les patients avec un PSA initial $<10 \mathrm{ng} / \mathrm{ml}$, et de $66 \%$ à $87 \%$ pour les patients avec un score de Gleason maximum à 6 . Le taux de biopsies négatives observé après curiethérapie varie selon les séries de $74 \%$ à $90 \%$ [39, 36, 33, 40, 41].

Après traitement par ultrasons, il n'y a pas de correspondance parfaite entre le taux de PSA et les biopsies (des cancers résiduels sont détectés chez $9 \%$ des patients dont le PSA nadir est inférieur à $0,5 \mathrm{ng} / \mathrm{ml}$ ) ; 1'utilisation de deux critères associés (PSA et biopsies de contrôle) permet une meilleure évaluation du statut carcinologique réel des patients après traitement par agents physiques.

Etant donné le nombre de patients traités par ultrasons et le recul modéré de l'étude (5 ans), il est trop tôt pour établir de véritables comparaisons avec les grandes séries publiées de patients traités par radiations ionisantes, d'ailleurs seule une étude randomisée incluant un très grand nombre de patients pourrait permettre une véritable comparaison.

\section{Retentissement sur la fonction sexuelle}

Le taux d'impuissance relativement élevé (64\%) observé chez les patients traités entre 1993 et 2001 doit être interprété en fonction de deux facteurs. Tout d'abord, l'âge moyen de la population est très élevé ( 72 ans), et les patients plus jeunes traités pendant cette période n'étaient pas candidats à la chirurgie en raison de tares associées. Par ailleurs, dans cette première série, la préservation de la fonction sexuelle n'a jamais été un objectif. Après ABLA-

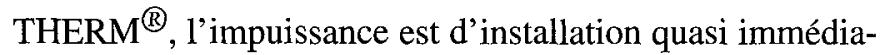
te car elle correspond à une brûlure des nerfs érecteurs et il est exceptionnel d'observer des récupérations tardives.

A la lumière des connaissances acquises depuis la modélisation informatique de la zone de nécrose, il est certainement possible, chez des patients sélectionnés (T1b-T1c), de réaliser une préservation des bandelettes neuro-vasculaires grâce à une répartition spatiale particulière des tirs au sein de l'apex prostatique. Il est cependant nécessaire pour protéger les bandelettes neuro-vasculaires, de ménager une marge de sécurité sur toute la hauteur de la prostate : le taux d'impuissance complète reste en effet élevé si la préservation n'est réalisée qu'au niveau de l'apex. Chaussy et Thuroff $[7,8]$ ont ainsi pu obtenir une préservation de la fonction érectile dans les deux tiers des cas en ménageant unilatéralement une marge de sécurité de $6 \mathrm{~mm}$ 
sur toute la hauteur de la prostate chez des patients dont les biopsies étaient positives dans un seul lobe prostatique ; cependant ils ont ensuite observé, chez les patients traités selon ce protocole, des biopsies de contrôle positives dans $15 \%$ des cas dans le lobe traité de façon partielle. Dans l'état actuel de nos connaissances, il existe donc pour le traitement par ultrasons le même dilemme que pour la chirurgie radicale : la préservation des nerfs érecteurs expose à un moins bon contrôle carcinologique. Cependant, la possibilité de répéter le traitement permet de compléter le contrôle carcinologique en cas de nécessité ; le risque d'impuissance après retraitement est alors majeur.

Les traitements par radiations ionisantes comportent également un risque de dysérection. Le taux d'impuissance après radiothérapie externe varie d'une série à l'autre de 11 à $67 \%[29,34,43,3,20,21,37,2,50,47,25,24,48,18$, $11,4,42,27,10,23]$. Des facteurs prédictifs de développement d'une impuissance après irradiation ont été retrouvés comme l'âge des patients, un traitement hormonal associé ou la notion d'une dysfonction érectile préalable. Cependant, il faut également noter que le taux d'impuissance après traitement augmente avec le suivi. Ainsi, Zelefsky note un taux d'impuissance de $28 \%$ à 2 ans et de 43 à $60 \%$ à 5 ans [48]. De même, Talcott [43] note un taux d'impuissance de $45 \%$ avant traitement et de 58 et $67 \%$ à 3 mois et à 1 an respectivement.

Après curiethérapie, le taux d'impuissance varie de 0 à $29 \%$ selon les études $[16,17,14,1,45,48,44,46,49,40$, 36]. Comme pour la radiothérapie externe, l'âge des patients est également un facteur prédictif de développement d'une impuissance. Ainsi, Ragde [33] note une préservation des fonctions érectiles de $90 \%$ avant 60 ans, de $80 \%$ de 60 à 70 ans et de $50 \%$ pour les patients de plus de 70 ans. Le taux d'impuissance est également plus important avec le suivi. Pour Stock [40], le taux d'impuissance est de $2,5 \%$ à 1 an et de $6 \%$ à 2 ans. Pour Zelefsky [49, 50], ce taux est de $21 \%$ à 2 ans et de $43 \%$ à 4 ans.

\section{CONCLUSION}

Le traitement du cancer localisé de la prostate par les ultrasons focalisés est une nouvelle option de traitement par agent physique qui présente plusieurs avantages :

- Absence d'irradiation ;

- Brève hospitalisation (< une semaine) ;

- Traitement réalisable sous anesthésie loco-régionale ;

- Le traitement peut être répété en cas de résultat incomplet ;

- Préservation possible de la fonction érectile chez les patients porteurs de cancer dépisté précocement (stade T1c).
Enfin, le traitement ABLATHERM n'est pas une impasse thérapeutique : en cas d'échec, le traitement par radiothérapie externe peut être proposé en "rattrapage" : il ne comporte aucun risque particulier et permet d'offrir au patient une option thérapeutique à visée curatrice "de seconde ligne".

\section{REFERENCES}

1. ARTERBERY V.E., WALLNER K., ROY J., FUKS Z. : Shortterm morbidity from CT-planned transperineal I-125 prostate implants. Int. J. Radiat. Oncol. Biol. Phys., 1993, 25 : 661-667.

2. BAGSHAW M.A., COX R.S., RAY G.R. : Status of radiation treatment of prostate cancer at Stanford University. NCI Monogr., 1988, $7: 47-60$.

3. BANKER F.L. : The preservation of potency after external beam irradiation for prostate cancer. Int. J. Radiat. Oncol. Biol. Phys., 1988, 15 : 219-220.

4. BEARD C.J., LAMB C., BUSWELL L. et al. : Radiation-associated morbidity in patients undergoing small-field external beam irradiation for prostate cancer. Int. J. Radiat. Oncol. Biol. Phys., 1998, $41:$ 257-262.

5. CHAPELON J.Y., MARGONARI J., THEILLERE Y. et al. : Effects of high-energy focused ultrasound on kidney tissue in the rat and the dog. Eur. Urol., 1992, $22: 147-152$.

6. CHAPELON J.Y., MARGONARI J., VERMIER F., GORY F., ECOCHARD R., GELET A. : In vivo effects of high intensity ultrasound on prostatic adenocarcinoma Dunning R3327. Cancer Res., 1992, $52:$ 6353-6457.

7. CHAUSSY C., THUROFF S. : High intensity focused ultrasound in prostate cancer : results after 3 years. Mol. Urol., 2000, $4: 179-182$.

8. CHAUSSY C., THUROFF S. : Results and side effects of High intensity focused ultrasound in localized prostate cancer. $\mathrm{J}$. Endourol., 2001, 15 : 437-440.

9. CHAVRIER F., CHAPELON J.Y., GELET A., CATHIGNOL D.: Modeling of high-intensity focused ultrasound-induced lesions in the presence of cavitation bubbles. J. Acoust. Soc. Am., 2000, 108: 432-440.

10. CHINN D.M., HOLLAND J., CROWNOVER R.L., ROACH M. III : Potency following high-dose three-dimensional conformal radiotherapy and the impact of prior major urologic surgical procedures in patients treated for prostate cancer. Int. J. Radiat. Oncol. Biol. Phys., 1995, 33 : 15-22.

11. CROOK J., ESCHE B., FUTTER N. : Effect of pelvic radiotherapy for prostate cancer on bowel, bladder, and sexual function : the patient's perspective. Urology, 1996, $47: 387-394$.

12. D'AMICO A.V., WHITTINGTON R., KAPLAN I. et al. : Equivalent biochemical failure-free survival after external beam radiation therapy or radical prostatectomy in patients with a pretreatment prostate specific antigen of $>4-20 \mathrm{ng} / \mathrm{ml}$. Int. J. Radiat. Oncol. Biol. Phys., 1997, 37 : 1053-1058.

13. D'AMICO A.V., WHITTINGTON R., MALKOWICZ S.B. et al. : Biochemical outcome after radical prostatectomy, external beam radiation therapy, or interstitial radiation therapy for clini- 
cally localized prostate cancer. J. Am. Med. Ass., 1998, 280 : 969-974.

14. D'AMICO A. : Radiation and hormonal therapy for locally advanced and clinically localized prostate cancer. Urology, 2001, $58: 78-82$.

15. FUKUNAGA-JOHNSON N., SANDLER H.M., MC LAUGHLIN P.W. et al. : Results of $3 \mathrm{D}$ conformal radiotherapy in the treatment of localized prostate cancer. Int. J. Radiat. Oncol. Biol. Phys., 1997, 38 : 311-317.

16. GELET A., CHAPELON J.Y., MARGONARI J. et al. : High intensity focused ultrasound : Experimentation on canine prostate. J. Endourol., 1993, $7: 249-253$.

17. GELET A., CHAPELON J.Y., MARGONARI J. et al. : Treatment of prostate cancer with transrectal focused ultrasound : early clinical experience. Eur. Urol., 1996, 29 : 174-183.

18. JONLER M., RITTER M.A., BRINKMANN R., MESSING E.M., RHODES P.R., BRUSKEWITZ R.C. : Sequelae of definitive therapy for prostate cancer localized to the pelvis. Urology, $1994,44: 876-882$.

19. KUPELIAN P., KATCHER J., LEVIN H. et al. : External beam radiotherapy versus radical prostatectomy for clinical stage T1$\mathrm{T} 2$ prostate cancer : Therapeutic implications of stratification by pretreatment PSA levels and biopsy Gleason scores. Cancer J. Sci. Am., 1997, 3 : 78-87.

20. LILLEBY W., FOSSA S.D., WAEHRE H.R., OLSEN D.R. : Long-term morbidity and quality of life in patients with localized prostate cancer undergoing definitive radiotherapy or radical prostatectomy. Int. J. Radiat. Oncol. Biol. Phys., 1999, 43 : 735-743.

21. LITWIN M.S., HAYS R.D., FINK A. et al. : Quality-of-life outcomes in men treated for localized prostate cancer. J. Am. Med. Ass., 1995, $273:$ 129-135.

22. LYONS J.A., KUPELIAN P.A., MOHAN D.S., REDDY C.A., KLEIN E.A. : Importance of high radiation doses $(72 \mathrm{~Gy}$ or greater) in the treatment of stage T1-T3 adenocarcinoma of the prostate. Urology, 2000, $55: 85-90$.

23. MADALINSKA J.B., ESSINK-BOT M.L., DE KONING H.J., KIRKELS W.J., VAN DER MAAS P.J. : Health-related qualityof-life effects of radical prostatectomy and primary radiotherapy for screen-detected or clinically diagnosed localized prostate cancer. J. Clin. Oncol., 2001, 19 : 1587-1588.

24. MANTZ C.A., SONG P., FARHANGI E. et al. : Potency probability following conformal megavoltage radiotherapy using conventional doses for localized prostate cancer. Int. J. Radiat. Oncol. Biol. Phys., 1997, 37 : 551-557.

25. NGUYEN L.N., POLLACK A., ZAGARS G.K. : Late effects after radiotherapy for prostate cancer in a randomized doseresponse study : results of a self-assessment questionnaire. Urology, 1998, 51 : 991-997.

26. PEARSON J.D., CARTER H.B. : Natural history of changes in prostate specific antigen in early stage prostate cancer. J. Urol., 1994, $152: 1743-1747$.

27. PEREZ C.A., LEE H.K., ANASATASIOS G., LOCKETT M.A.: Technical factors affecting morbidity in definitive irradiation for localized carcinoma of the prostate. Int. J. Radiat. Oncol. Biol.
Phys., 1994, $28: 811-819$.

28. PISANSKY T.M., KAHN M.J., BOSTWICK D.G. : An enhanced prognostic system for clinically localized carcinoma of the prostate. Cancer, 1997, $79: 2154-2161$.

29. POTOSKY A.L., LEGLER J., ALBERTSEN P.C. et al. : Health outcomes after prostatectomy or radiotherapy for prostate cancer: results from the Prostate Cancer Outcomes Study. J. Natl. Cancer Inst., 2000, 92 : 1582-1592.

30. POTTERS L., CHA C., OSHINSKY G., VENKATRAMAN E., ELEFSKY M., LEIBEL S. : Risk profiles to predict PSA relapse-free survival for patients undergoing permanent prostate brachytherapy. Cancer J. Sci. Am., 1995, 5 : 301-306.

31. PRESTIDGE B.R., HOAK D.C., GRIMM P.D., RAGDE H., CAVANAGH W., BLASKO J.C. : Posttreatment biopsy results following interstitial brachytherapy in early-stage prostate cancer. Int. J. Radiat. Oncol. Biol. Phys., 1997, 37 : 31-39.

32. RAGDE H., ELGAMAL A.A., SNOW P.B. et al. : Ten-year disease free survival after transperineal sonography-guided iodine125 brachytherapy with or without 45 -gray external beam irradiation in the treatment of patients with clinically localized, low to high Gleason grade prostate carcinoma. Cancer, 1998, 83 : 989-1001.

33. RAGDE H., BLASKO J.C., GRIMM P.D. et al. : Interstitial iodine-125 radiation without adjuvant therapy in the treatment of clinically localized prostate carcinoma. Cancer, 1997, 80 : $442-453$.

34. ROACH M. III, CHINN D.M., HOLLAND J., CLARKE M. : A pilot survey of sexual function and quality of life following $3 \mathrm{D}$ conformal radiotherapy for clinically localized prostate cancer. Int. J. Radiat. Oncol. Biol. Phys., 1996, 35 : 869-874.

35. ROUVIERE O., LYONNET D., RAUDRANT A. et al. : MRI Appearance of prostate following Transrectal HIFU ablation of localized cancer. Eur. Urol., 2001, $40: 265-274$.

36. SHARKEY J., CHOVNICK S.D., BEHAR R. et al. : Outpatient ultrasound-guided palladium 103 brachytherapy for localized adenocarcinoma of the prostate : a preliminary report of 434 patients. Urology, 1998, $51:$ 796-803.

37. SHIPLEY W.U., PROUT G.R. Jr., COACHMAN N.M. et al. : Radiation therapy for localized prostate carcinoma : Experience at the Massachusetts General Hospital (1973-1981). Natl. Cancer Inst. Monogr., 1988, $7: 67-73$.

38. STOCK R.G., STONE N.N. : The effect of pronostic factors on therapeutic outcome following transperineal prostate brachytherapy. Sem. Surg. Oncol., 1997, $13: 454-460$.

39. STOCK R.G., STONE N.N., KAO J., IANNUZZI C., UNGER P. : The effect of disease and treatment-related factors on biopsy results after prostate brachytherapy. Cancer, 2000, 89 : 18291834.

40. STOCK R.G., STONE N.N., DE WYNGAERT J.K., LAVAGNINI P., UNGER P.D. : Prostate specific antigen findings and biopsy results following interactive ultrasound guided transperineal brachytherapy for early stage prostate carcinoma. Cancer, $1996,77: 2386-2392$.

41. STOKES S.H. : Comparison of biochemical disease-free survival of patients with localized carcinoma of the prostate under- 
going radical prostatectomy, transperineal ultrasound-guided radioactive seed implantation, or definitive external beam irradiation. Int. J. Radiat. Oncol. Biol. Phys., 2000, 47 : 129-136.

42. TALCOTT J.A., RIEKER P., PROPERT K.J. et al. : Long-term complications of treatment for early prostate cancer : 2-year follow-up in a prospective multi-institutional outcomes study : Proceedings of the $32^{\text {nd }}$ meeting of ASCO. J. Clin. Oncol., 1996, $15: 252$ (Abstr).

43. TALCOTT J.A., RIEKER P., CLARK J.A. : Patient-reported symptoms after primary therapy for early prostate cancer : Results of a prospective cohort study. J. Clin. Oncol., 1998, 16 : 275-283.

44. WALLNER K., LEE H., WASSERMAN S., DATTTOLI M. : Low risk of urinary incontinence following prostate brachytherapy in patients with a prior transurethral prostate resection. Int. J. Radiat. Oncol. Biol. Phys., 1997, 37 : 565-569.

45. WALLNER K., ROY J., ZELEFSKY M., FUKS Z., HARRISON L. : Short-term freedom from disease progression after I125 prostate implantation. Int. J. Radiat. Oncol. Biol. Phys., 1994, $30: 405-409$.

46. WALLNER K., ROY J., HARRISON L. : Tumour control and morbidity following transperineal iodine 125 implantation for stage T1/T2 prostatic carcinoma. J. Clin. Oncol., 1996, 14 : 449453.

47. WILDER R.B., CHOU R.H., RYU J.K. et al. : Potency preservation after three-dimensional conformal radiotherapy for prostate cancer. Am. J. Clin. Oncol., 2000, 23 : 330-333.

48. ZELEFSKY M.J., WALLNER K.E., LING C.C. et al. : Comparison of the 5-year outcome and morbidity of three-dimensional conformal radiotherapy versus transperineal permanent iodine125 implantation for early-stage prostatic cancer. J. Clin. Oncol., 1999, $17: 517-522$.

49. ZELEFSKY M.J., HOLLISTER T., RABEN A., MATTHEWS S., WALLNER K.E. : Five-year biochemical outcome and toxicity with transperineal CT-planned permanent I-125 prostate implantation for patients with localized prostate cancer. Int. J. Radiat. Oncol. Biol. Phys., 2000, 47 : 1261-1266.

50. ZELEFSKY M.J., COWEN D., FUKS Z. et al. : Long term tolerance of high dose three-dimensional conformal radiotherapy in patients with localized prostate carcinoma. Cancer, 1999, 85 : 2460-2468.

\section{ABSTRACT}

High intensity focused ultrasound for the treatment of localized prostate cancer: efficacy and impact on sexual function

Albert GELET, Laura POISSONNIER, Jean-Yves CHAPELON, Raymonde BOUVIER, Olivier ROUVIERE,

Denis LYONNET, Jean Michel DUBERNARD.

Introduction: Since 1999, a therapeutic device using High Intensity Focused Ultrasound (HIFU) technology has been marketed in Europe for the treatment of localized prostate cancer. Clinical and technical development was designed to provide a minimally invasive alternative for these patients. The purpose of this study was to evaluate the efficacy of HIFU therapy for localized prostate cancer and its impact on sexual function.

Material and Methods: HIFU technology is based on a convergent beam of high intensity ultrasound that creates a sudden and sharp increase in temperature $\left(85^{\circ} \mathrm{C}\right.$ to $\left.100^{\circ} \mathrm{C}\right)$ in the tissues at the focal point. This leads to a precise lesion in the tissue, adjustable from 19 to $24 \mathrm{~mm}$ in height and $2 \mathrm{~mm}$ in diameter. Successive displacements of the focal point are computer-driven, allowing treatment of a defined volume. All patients were treated with the ABLATHERM ${ }^{8}$ device (EDAP SA, France); they were treated using the device prototypes between 1993 to 1999 and then with the marketed machine. The treatment procedure was improved from 2000 onwards with the combination of transurethral resection of the prostate (TURP) in order to reduce post-treatment catheter time. A nerve-sparing procedure was also tested in 2002.

The selected population included 120 patients considered to be potentially curable with clinical stage T1-T2 prostate cancer and an initial PSA $<10$ $\mathrm{ng} / \mathrm{ml}$ (group 1). A larger group of 167 patients with an initial PSA $<30 \mathrm{ng} / \mathrm{ml}$ was also considered (group 2). All patients were not candidates for surgery due to their age or comorbidities. In the two groups, clinical failure was defined by the need for administration of an adjuvant prostate cancer treatment (hormone deprivation or external radiation). Disease progression, or biochemical failure, was strictly defined as any evidence of residual cancer on follow-up biopsies (regardless of the PSA level), or 3 successive increases of the PSA level (with negative follow-up biopsies), with a velocity $>0.75$ $\mathrm{ng} / \mathrm{ml} /$ year. Disease-free survival rates were calculated using the Kaplan-Meier method. Survival rates were compared using the log-rank test. The impact 
of HIFU treatment on sexual function was assessed by a questionnaire in 70 patients who underwent standard HIFU treatment and in 28 patients in whom a nerve-sparing procedure was performed.

Results: Patient baseline characteristics ( \pm SD) were, in group 1 and group 2 respectively: mean age: $71.2( \pm 5.34)$ years and $71.8( \pm 5.11)$ years; clinical stage: T1 for 61 patients and T2 for 59 patients in group 1, and T1 for 77 patients, T2 for 85 patients and T3 for 5 patients in group 2; mean initial PSA level: $5.67( \pm 2.47) \mathrm{ng} / \mathrm{ml}$ and $9.30( \pm 6.01) \mathrm{ng} / \mathrm{ml}$; Gleason score: 2-6 for 77 patients and 7-10 for 43 patients in group 1, and 2-6 for 98 patients, 7 for 44 patients, and 8-10 for 25 patients in group 2; mean prostate volume: $33.6( \pm 16.5) \mathrm{ml}$ and $34.4( \pm 16.7)$ $\mathrm{ml}$, respectively. Mean follow-up was 27 months (range: 3-96 months) in group 1, and 23 months (range: 3-90 months) in group 2 . In group 1, a residual cancer was diagnosed in 17 patients, but only 6 patients needed adjuvant treatment due to a significant rise of the PSA level (hormone deprivation: $n=2$, external radiation: $n=4$ ), leading to a clinical success rate of $95 \%$. Similarly, in group 2, 36 patients presented with positive follow-up biopsies, and 21 of them required adjuvant treatment (hormone deprivation: $n=10$, external radiation: $n=11$ ), leading to a clinical success rate of $87.5 \%$. The disease-free survival rates (previously defined on the combined biopsy and PSA criteria) were $76.9 \%$ and $66 \%$ in group 1 and 2 , respectively. In addition, the disease-free survival rate in group 2 was stratified according to the initial prognosis risk level: $85 \%$ in low-risk patients (i.e. patients with clinical stage T1T2a and PSA $<10 \mathrm{ng} / \mathrm{ml}$ and Gleason score < 7), $67.5 \%$ in intermediate-risk patients (i.e. clinical stage T2b or PSA $10-20 \mathrm{ng} / \mathrm{ml}$ or Gleason score $=7$ ), and $42 \%$ in high-risk patients (i.e. clinical stage T2C or PSA $>20 \mathrm{ng} / \mathrm{ml}$ or Gleason score $>7$ ). In the overall population, 70 patients had normal sexual function prior to HIFU treatment; 25 patients $(36 \%)$ still had erections allowing sexual intercourse with penetration after treatment. A nerve-sparing procedure was also performed in 28 potent patients: $\mathbf{4 3} \%$ of these patients had persistent erections allowing sexual intercourse with penetration after treatment, indicating that this nerve-sparing procedure still needs to be improved.
Conclusion: The efficacy results observed after HIFU treatment are similar to those observed after other non-surgical treatments for prostate cancer. After complete HIFU treatment of the gland, more than $1 / 3$ of patients still reported erections allowing sexual intercourse with penetration; these results must be interpreted for an elderly population (mean age: 72 years). A nerve-sparing procedure is currently being perfected and tested.

Key words: focused ultrasound, localized prostate cancer, erectile dysfunction 